\title{
EFICÁCIA DO GLYPHOSATE E 2,4-D NO CONTROLE DE COMMELINA VILLOSA
}

\author{
C.F. de Campos ${ }^{1}$, A.C.P. Rodrigues-Costa ${ }^{2}$, L.A. Cardoso ${ }^{1}$, M.R.R. Pereira ${ }^{1}$, G.S.F. de Souza ${ }^{1}$, D. Martins ${ }^{1}$ \\ ${ }^{1}$ Universidade Estadual Paulista, Faculdade de Ciências Agronômicas, Fazenda Lageado, CP 237, CEP \\ 18610-307, Botucatu, SP, Brasil. E-mail: caio.agro@hotmail.com
}

\section{RESUMO}

\begin{abstract}
O objetivo do presente trabalho foi avaliar a eficácia de glyphosate e2,4-D, isolados e em mistura, no controle de Commelina villosa. Foram estudadas duas metodologias de avaliação de absorção de herbicidas em oito intervalos de tempo para a lavagem (simulando chuva após a aplicação) e corte (simulando abortamento, como estratégia de defesa) das folhas: 2, 4, 6, 8, 12, 24 e 48 horas após a aplicação dos herbicidas, além de um tratamento sem lavagem ou corte das folhas, em delineamento experimental inteiramente casualizado com quatro repetições, dispostos em um esquema fatorial $3 \times 7+1$ (três herbicidas $x$ sete períodos - horas após a aplicação). Os herbicidas e doses testados foram: glyphosate $\left(1.440 \mathrm{~g} \mathrm{ha}^{-1}\right), 2,4-\mathrm{D}\left(720 \mathrm{~g} \mathrm{ha}^{-1}\right)$ e a mistura glyphosate $+2,4-\mathrm{D}$ $\left(1.080+720 \mathrm{~g} \mathrm{ha}^{-1}\right)$. A simulação de chuva interferiu de forma negativa no controle das plantas com o herbicida glyphosate. O controle com o herbicida 2,4-D foi influenciado apenas no período de 2 horas. Os períodos de simulação de chuva não influenciaram no controle das plantas com a mistura de glyphosate + 2,4-D. Para o estudo com corte das folhas tratadas, todos os tratamentos independente do período para corte das folhas foram influenciados de forma negativa no controle, sendo que as plantas apresentaram rebrotas quando tratadas com o herbicida 2,4-D isolado.
\end{abstract}

PALAVRAS-CHAVE: Trapoeraba, Commelinaceae, herbicida.

\section{ABSTRACT}

EFFICACY OF GLYPHOSATE AND 2,4-D IN THE CONTROL OF COMMELINA VILLOSA. The aim of this study was to evaluate the efficacy of glyphosate and 2,4-D alone and in combination, in the control of Commelina villosa. We studied two methodologies for evaluating herbicide absorption in eight time intervals for washing (simulating rainfall after application) and cutting of leaves (simulating abortion as a defense strategy): 2, 4, 6, 8, 12, 24 and 48 hours after herbicide application, and a treatment without washing or cutting the leaves in a completely randomized design with four replications in a $3 \times 7+1$ factorial design (three herbicides $\times$ seven periods - hours after application). Herbicides and doses tested were: glyphosate $\left(1,440 \mathrm{~g} \mathrm{ha}^{-1}\right), 2,4-\mathrm{D}\left(720 \mathrm{~g} \mathrm{ha}^{-1}\right)$ and a mixture of glyphosate $+2,4-\mathrm{D}\left(1,080+720 \mathrm{~g} \mathrm{ha}^{-1}\right)$. The simulation of rain interfered negatively in the plant control with glyphosate. The control with the herbicide 2,4-D was affected only for the period of 2 hours. Periods of rain simulation did not influence the control of plants with a mixture of glyphosate $+2,4-\mathrm{D}$. For the study with the cutting of treated leaves, all treatments regardless of the period of cutting the leaves were influenced negatively in terms of plant control, the plants showing regrowth when treated with 2,4-D alone.

KEY WORDS: Dayflower, Commelinaceae, herbicide.

\section{INTRODUÇÃO}

O glyphosate é recomendado para controle de diversas plantas daninhas, mas no caso de trapoerabas (Commelina spp.), muitas vezes não ocorre um controle satisfatório. Entre as espécies de trapoeraba que têm apresentado tolerância às aplicações de glyphosate, destacam-se a C. benghalensis, C. diffusa, C. erecta e C. nudiflora (TuFri SANTOS et al., 2004; Rocha et al., 2007; MACIEL, et al., 2011). Por isso, a mistura do glyphosate com outros herbicidas tem se tornado prática comum, sendo 2,4-D o mais utilizado, embora, em determinadas condições, seja muito tóxico à cultura (SANTOS et al., 2002).

Sullivan; Donovan (2006) relataram que o uso da mistura de glyphosate $+2,4$-D aumenta a porcentagem de controle das espécies daninhas, pois esses herbicidas danificam os vasos condutores e afetam a translocação dos produtos na planta. Devido ao provável sinergismo entre os produtos, a mistura glyphosate + 2,4-D também foi indicada por ToLLERvEY et al. (1979) para controlar trapoeraba em diversas

${ }^{2}$ Universidade Estadual do Oeste do Paraná, Cascavel, PR, Brasil. 
culturas. GALLI (1991) estudando o controle de C. virginica em pomares de citros obteve um melhor resultado com a mistura de glyphosate $+2,4$-D do que a aplicação isolada dos produtos, da mesma forma que RAmOs; DuRigan (1996) ao utilizarem glyphosate e2,4-D amina, aplicados isoladamente ou em mistura, também constataram que a aplicação da mistura apresentou o melhor controle de C. virginica na cultura de citros.

Adiferença na suscetibilidade de plantas daninhas a herbicidas têm sido atribuída ao estádio de desenvolvimento da planta, à morfologia (área e forma do limbo, ângulo ou orientação das folhas em relação ao jato de pulverização), a anatomia foliar (presença de estômatos na superfície adaxial, presença de pêlos, espessura e composição da camada cuticular) e a diferença na absorção, translocação, compartimentalização e metabolismo da molécula herbicida (VARGAS et al., 1999). Segundo WILSON (1981), a dificuldade de controle de espécies da família Commelinaceae pode ser atribuída ao duplo mecanismo de reprodução que elas apresentam: por sementes e por enraizamento dos nós. Para VARGAs et al. (1999) a tolerância das trapoerabas aos herbicidas pode estar relacionada com a não-sensibilidade da enzima EPSPs (5 enolpiruvilshikimato-3-fosfato sintase) aos herbicidas.

SegundoSHERMAN etal.(1996), existem pelo menos quatro mecanismos gerais que podem explicar a resistência e/ou tolerância de uma planta a herbicidas: redução da concentração do herbicida no local de ação; absorção foliar e/ou translocação reduzida do herbicida; metabolizaçãoe/ou destoxificação intensa do herbicida a substâncias menos fitotóxicas e perda de afinidade do herbicida pelo local de ação.

Assim, as hipóteses deste trabalho baseiam-se no fato de que as diferenças no controle de espécies de Commelinas encontradas na literatura podem estar relacionadas aos processos de absorção dos herbicidas utilizados, e que o abortamento das partes vegetativas com injúrias de fitointoxicação apresenta-se como estratégia da planta em impedir a translocação. Desta forma, o uso de técnicas alternativas como a lavagem e/ou o corte da folha em diferentes períodos após a aplicação do herbicida podem auxiliar na compreensão das diferenças de tolerância entre as espécies.

O objetivo do presente trabalho foi avaliar períodos de absorção de glyphosate e 2,4-D, isolados e em mistura, no controle de plantas de Commelina villosa.

\section{MATERIAL E MÉTODOS}

O estudo com plantas de C. villosa foi conduzido no Núcleo de Pesquisas Avançadas em Matologia (NUPAM), da FCA/UNESP, campus de Botucatu, $\mathrm{SP}$, em condições de casa de vegetação. Das plantas matrizes, foram retiradas segmentos de caule de aproximadamente $15 \mathrm{~cm}$ de comprimento, contendo três nós, o qual foi plantado um segmento de caule por vaso, enterrando-se um nó. As mudas foram transplantadas em vasos de plástico, com capacidade para 2,5 L, preenchidos com substrato composto por solo, adubo químico (4-14-8) e orgânico.

$\mathrm{O}$ experimento foi instalado em delineamento experimental inteiramente casualizado em esquema fatorial $3 \times 7+1$ (três herbicidas $\times$ sete períodos - horas após a aplicação) utilizando-se duas metodologias de avaliação de absorção de herbicidas (simulação de chuva e corte das folhas aplicadas), além de uma testemunha sem aplicação, com quatro repetições. Os tratamentos consistiram de oito intervalos de tempo para lavagem (simulando chuva após a aplicação) e para o corte (simulando abortamento, como estratégia de defesa) das folhas aplicadas: 2, 4, 6, 8, 12, 24 e 48 horas após a aplicação dos herbicidas, além de um tratamento sem lavagem ou corte das plantas. Após esses intervalos as folhas aplicadas foram retiradas por meio do corte dos ramos ou lavadas por meio de uma simulação de chuva de $20 \mathrm{~mm}$, realizada em um simulador de pulverização estacionário.

Foram aplicados os herbicidas glyphosate (1.440 g e.a. ha ${ }^{-1}$ - Produto comercial, Glifosato Atanor), 2,4-D amina (720 g e.a. ha ${ }^{-1}$ - Produto comercial, U46 D-Fuid 2,4-D) e a mistura glyphosate + 2,4-D (1.080 +720 g e.a. ha-1).

Para o estudo com simulação de chuva, os herbicidas foram aplicados utilizando-se um pulverizador costal, pressurizado a $\mathrm{CO}_{2}$ e munido de barra com quatro pontas de jato plano XR 11002 VS, espaçadas entre si de $50 \mathrm{~cm}$, com pressão de $200 \mathrm{kPa}$ e consumo de calda de $200 \mathrm{~L} \mathrm{ha}^{-1}$. Os herbicidas foram aplicados quando as plantas tinham de 3 a 5 ramos com 6 a 8 folhas completamente expandidas A simulação de chuva de $20 \mathrm{~mm}$, suficiente para a lixiviação do produto (ForNAROLl et al. 1997), foi realizada em um simulador de pulverização estacionário. No momento da chuva os vasos foram envolvidos por sacos de plástico, evitando que os herbicidas entrassem em contato com o solo.

Para o estudo com o corte (simulando abortamento como estratégia de defesa) das folhas tratadas, antes da aplicação dos herbicidas, determinou-se a capacidade de retenção de volume decalda das folhas C. villosa, utilizando-se a metodologia proposta por SouzA et al. (2007). A plicou-se 11,0 uL folha-1 ${ }^{-1}$, sendo que as plantas tinham de 3 a 5 ramos com 6 a 8 folhas completamente expandidas, sendo depositado sobre a face adaxial o volume pré-determinado na terceira folha a partir do ápice a qual foi cortada de acordo com os intervalos de tempo de cada tratamento. A aplicação foi realizada com o auxílio de uma micropipeta, evitando o escorrimento da calda nas folhas aplicadas. 
Após a aplicação dos herbicidas, as plantas retornaram para a casa de vegetação, onde aguardaram os respectivos intervalos de tempo propostos nos tratamentos, tanto para a simulação de chuva quanto para o corte das folhas.

As avaliações visuais de controle foram realizadas aos 7, 14, 21 e 28 dias após a aplicação dos tratamentos (DAA), através de uma escala de percentual de notas, no qual 0 (zero) correspondeu a nenhuma injúria e 100 (cem) a morte das plantas, segundo critérios da SOCIEDADE... (1995). Os parâmetros utilizados para estabelecimento das notas foram: acúmulo de biomassa, inibição do crescimento, quantidade e uniformidade das injurias, capacidade de rebrota das plantas.

Os resultados foram submetidos à análise de variância pelo Teste " $\mathrm{F}$ " e as médias dos tratamentos foram comparadas pelo teste de Tukey a $5 \%$ de probabilidade.

\section{RESULTADOS E DISCUSSÃO}

Observa-se que, independentemente do período de avaliação de controle das plantas, quando estas foram lavadas ou cortadas, houve interação significa- tiva pelo teste $\mathrm{F}$ entre os diferentes fatores analisados (Tabelas 1 a 8 ).

Verifica-se que quando da aplicação do herbicida glyphosate na forma isolada, este proporcionou os menores níveis de controle aos 7, 14, 21 e 28 DAA independente dos períodos de simulação de chuva testados, com exceção aos 7 DAA em que a simulação de chuva ocorreu 2 horas após a aplicação, em que apresentou comportamento semelhante aos demais tratamentos (Tabelas 1 a 4). Contudo, não foi observado diferença no controle das plantas com o herbicida glyphosate independente dos períodos de simulação de chuva e de avaliação.

Para a aplicação de glyphosate na forma isolada, o controle das plantas só foi considerado excelente a partir de 21 DAA quando as plantas não receberam a simulação de chuva, sendo esse controle acima de $90 \%$. Esses resultados indicam que o processo de absorção do glyphosate isolado ocorre de forma relativamente lenta, e aumenta com o passar do tempo, sendo que a simulação de chuva independente do período influencia de forma negativa no controle das plantas, disponibilizando menor quantidade de molécula do herbicida para ser absorvida pela planta.

Tabela 1 - Porcentagem de controle das plantas de Commelina villosa, aos sete dias após aplicação, em função de períodos de simulação de chuva. Botucatu, SP, 2009.

\begin{tabular}{|c|c|c|c|c|c|c|c|c|c|}
\hline \multirow[b]{2}{*}{ Tratamento } & \multirow{2}{*}{$\begin{array}{l}\text { Dose } \\
\left(\mathrm{g} \mathrm{ha}^{-1}\right)\end{array}$} & \multicolumn{8}{|c|}{ Períodos (horas) } \\
\hline & & 2 & 4 & 6 & 8 & 12 & 24 & 48 & $\begin{array}{c}\text { Sem } \\
\text { lavagem }\end{array}$ \\
\hline$\overline{\text { Glyphosate }}$ & 1.440 & $12,50 \mathrm{aA}$ & $13,25 \mathrm{bA}$ & $11,50 \mathrm{bA}$ & $13,25 \mathrm{bA}$ & $11,75 \mathrm{bA}$ & $11,00 \mathrm{bA}$ & $11,25 \mathrm{cA}$ & $15,00 \mathrm{cA}$ \\
\hline $2,4-\mathrm{D}$ & 720 & $17,00 \mathrm{aCD}$ & $9,75 b D$ & 18,75abBCD & $22,50 \mathrm{aBC}$ & $21,25 \mathrm{aBC}$ & $28,75 \mathrm{aB}$ & $62,00 \mathrm{aA}$ & $60,00 \mathrm{aA}$ \\
\hline Glyphosate+2,4-D & $1.080+720$ & $20,00 \mathrm{aC}$ & $23,75 \mathrm{aABC}$ & $21,25 \mathrm{aBC}$ & $25,00 \mathrm{aABC}$ & $26,25 \mathrm{aABC}$ & $26,25 \mathrm{aABC}$ & $31,50 \mathrm{bAB}$ & $33,25 \mathrm{bA}$ \\
\hline $\mathrm{F}_{\text {Herbicida }}(\mathrm{H})$ & & & & & $117,209^{* *}$ & & & & \\
\hline $\mathrm{F}_{\text {Chuva }}^{\text {Herbicida }}(\mathrm{C})$ & & & & & $34,447^{* *}$ & & & & \\
\hline $\mathrm{F}(\mathrm{H}) \times \mathrm{F}(\mathrm{C})$ & & & & & $19,020^{* *}$ & & & & \\
\hline CV $(\%)$ & & & & & 21,1 & & & & \\
\hline Dms (H) & & & & & 8,13 & & & & \\
\hline Dms (C) & & & & & 10,60 & & & & \\
\hline
\end{tabular}

**significativo a 1\% de probabilidade. Médias seguidas de mesma letra minúscula na coluna e maiúscula na linha, não diferem estatisticamente entre si, pelo teste de tukey $(\mathrm{P}>0,05)$.

Tabela 2 - Porcentagem de controle das plantas de Commelina villosa, aos quatorze dias após aplicação, em função de períodos de simulação de chuva. Botucatu, SP, 2009.

\begin{tabular}{|c|c|c|c|c|c|c|c|c|c|}
\hline \multirow{2}{*}{ Tratamento } & \multirow{2}{*}{ Dose $\left(\mathrm{g} \mathrm{ha}^{-1}\right)$} & \multicolumn{8}{|c|}{ Períodos (horas) } \\
\hline & & 2 & 4 & 6 & 8 & 12 & 24 & 48 & Sem lavagem \\
\hline Glyphosate & 1.440 & $30,00 \mathrm{bB}$ & $35,25 \mathrm{cB}$ & $25,50 \mathrm{bB}$ & $37,25 \mathrm{bB}$ & $32,50 \mathrm{bB}$ & $28,75 \mathrm{bB}$ & $35,00 \mathrm{bB}$ & $62,50 \mathrm{bA}$ \\
\hline 2,4-D & 720 & $71,00 \mathrm{aB}$ & $73,75 \mathrm{bB}$ & $87,50 \mathrm{aAB}$ & $90,00 \mathrm{aAB}$ & $100,00 \mathrm{aA}$ & $100,00 \mathrm{aA}$ & $100,00 \mathrm{aA}$ & $100,00 \mathrm{aA}$ \\
\hline Glyphosate+2,4-D & $1.080+720$ & $82,50 \mathrm{aA}$ & $92,50 \mathrm{aA}$ & $93,75 \mathrm{aA}$ & $93,75 \mathrm{aA}$ & $95,00 \mathrm{aA}$ & $95,00 \mathrm{aA}$ & $93,75 \mathrm{aA}$ & $93,75 \mathrm{aA}$ \\
\hline $\mathrm{F}_{\text {Herbicida }}(\mathrm{H})$ & & & & & $301,511^{* *}$ & & & & \\
\hline $\mathrm{F}_{\text {Chuva }}(\mathrm{C})$ & & & & & $5,713^{* *}$ & & & & \\
\hline $\mathrm{F}(\mathrm{H}) \times \mathrm{F}(\mathrm{C})$ & & & & & $2,516^{* *}$ & & & & \\
\hline $\mathrm{CV}(\%)$ & & & & & 14,3 & & & & \\
\hline Dms $(\mathrm{H})$ & & & & & 17,70 & & & & \\
\hline Dms (C) & & & & & 23,09 & & & & \\
\hline
\end{tabular}

**significativo a 1\% de probabilidade. Médias seguidas de mesma letra minúscula na coluna e maiúscula na linha, não diferem estatisticamente entre si, pelo teste de tukey $(\mathrm{P}>0,05)$. 
Tabela 3 - Porcentagem de controle das plantas de Commelina villosa, aos 21 dias após aplicação, em função de períodos de simulação de chuva. Botucatu, SP, 2009.

\begin{tabular}{|c|c|c|c|c|c|c|c|c|c|}
\hline \multirow{2}{*}{ Tratamento } & \multirow{2}{*}{ Dose $\left(\mathrm{g} \mathrm{ha}^{-1}\right)$} & \multicolumn{8}{|c|}{ Períodos (horas) } \\
\hline & & 2 & 4 & 6 & 8 & 12 & 24 & 48 & Sem lavagem \\
\hline Glyphosate & 1.440 & $57,25 \mathrm{bC}$ & $75,75 \mathrm{bB}$ & $71,25 \mathrm{bBC}$ & $79,50 \mathrm{bB}$ & $68,25 \mathrm{bBC}$ & $75,00 \mathrm{bB}$ & $76,50 \mathrm{bB}$ & $96,25 \mathrm{aA}$ \\
\hline $2,4-\mathrm{D}$ & 720 & $87,50 \mathrm{aA}$ & $88,25 \mathrm{aA}$ & $96,50 \mathrm{aA}$ & $96,25 a A$ & $100,00 \mathrm{aA}$ & $100,00 \mathrm{aA}$ & $100,00 \mathrm{aA}$ & $100,00 \mathrm{aA}$ \\
\hline Glyphosate+2,4-D & $1.080+720$ & $87,50 \mathrm{aA}$ & $96,25 \mathrm{aA}$ & $97,50 \mathrm{aA}$ & $100,00 \mathrm{aA}$ & $100,00 \mathrm{aA}$ & $100,00 \mathrm{aA}$ & $100,00 \mathrm{aA}$ & $100,00 \mathrm{aA}$ \\
\hline $\mathrm{F}_{\text {Herbicida }}(\mathrm{H})$ & & & & & $103,630^{* *}$ & & & & \\
\hline $\mathrm{F}_{\text {Chuva }}(\mathrm{C})$ & & & & & $8,831^{* *}$ & & & & \\
\hline $\mathrm{F}(\mathrm{H}) \times \mathrm{F}(\mathrm{C})$ & & & & & $2,682^{* *}$ & & & & \\
\hline $\mathrm{CV}(\%)$ & & & & & 7,7 & & & & \\
\hline Dms $(\mathrm{H})$ & & & & & 11,60 & & & & \\
\hline Dms (C) & & & & & 15,12 & & & & \\
\hline
\end{tabular}

** significativo a 1\% de probabilidade. Médias seguidas de mesma letra minúscula na coluna e maiúscula na linha, não diferem estatisticamente entre si, pelo teste de tukey $(\mathrm{P}>0,05)$.

Tabela 4 - Porcentagem de controle das plantas de Commelina villosa, aos 28 dias após aplicação, em função de períodos de simulação de chuva nas plantas. Botucatu, SP, 2009.

\begin{tabular}{|c|c|c|c|c|c|c|c|c|c|}
\hline \multirow{2}{*}{ Tratamento } & \multirow{2}{*}{ Dose $\left(\mathrm{g} \mathrm{ha}^{-1}\right)$} & \multicolumn{8}{|c|}{ Períodos (horas) } \\
\hline & & 2 & 4 & 6 & 8 & 12 & 24 & 48 & Sem lavagem \\
\hline Glyphosate & 1.440 & $53,75 \mathrm{bB}$ & $62,50 \mathrm{bB}$ & $71,50 \mathrm{bB}$ & $71,50 \mathrm{bB}$ & $66,50 \mathrm{bB}$ & $70,00 \mathrm{bB}$ & $70,00 \mathrm{bB}$ & $98,25 \mathrm{aA}$ \\
\hline $2,4-\mathrm{D}$ & 720 & $81,25 \mathrm{aB}$ & $86,25 \mathrm{aAB}$ & $99,75 \mathrm{aA}$ & $98,75 \mathrm{aAB}$ & $100,00 \mathrm{aA}$ & $100,00 \mathrm{aA}$ & $100,00 \mathrm{aA}$ & $100,00 \mathrm{aA}$ \\
\hline Glyphosate+2,4-D & $1.080+720$ & $93,25 \mathrm{aA}$ & $99,75 \mathrm{aA}$ & $100,00 \mathrm{aA}$ & $100,00 \mathrm{aA}$ & $100,00 \mathrm{aA}$ & $100,00 \mathrm{aA}$ & $100,00 \mathrm{aA}$ & $100,00 \mathrm{aA}$ \\
\hline $\mathrm{F}_{\text {Herbicida }}(\mathrm{H})$ & & & & & $120,018^{* *}$ & & & & \\
\hline $\mathrm{F}_{\text {Chuva }}(\mathrm{C})$ & & & & & $8,308^{* *}$ & & & & \\
\hline $\mathrm{F}(\mathrm{H}) \times \mathrm{F}(\mathrm{C})$ & & & & & $2,722^{* *}$ & & & & \\
\hline $\mathrm{CV}(\%)$ & & & & & 9,13 & & & & \\
\hline Dms (H) & & & & & 13,67 & & & & \\
\hline Dms (C) & & & & & 17,83 & & & & \\
\hline
\end{tabular}

** significativo a 1\% de probabilidade. Médias seguidas de mesma letra minúscula na coluna e maiúscula na linha, não diferem estatisticamente entre si, pelo teste de tukey $(\mathrm{P}>0,05)$.

Para a aplicação do herbicida 2,4-D na forma isolada ou a mistura de glyphosate $+2,4-\mathrm{D}$, proporcionaram controle semelhante, exceto para a aplicação de 2,4-D na forma isolada no intervalo de 4 horas de simulação de chuva aos 7 e 14 DAA, onde esse controle foi inferior ao da mistura glyphosate $+2,4$-D. Nota-se que tanto 2,4-D isolado quanto sua mistura com glyphosate proporcionaram controle acima de $70 \%$ a partir de 14 DAA. Contudo aos 14 DAA o tratamento com 2,4-D na forma isolada atingiu controle de $100 \%$ a partir do intervalo de 12 horas de simulação de chuva (Tabelas 1 a 4 ).

MONQUEROetal. (2004a) verificaram uma absorção lenta do glyphosate até 24 horas após a aplicação em C. benghalensis, já o presente estudo mostrou não haver diferença na absorção até 48 horas para o mesmo herbicida, sendo assim mais lenta para C.villosa que para C. benghalensis. Em outro estudo, MONQUERO et al. (2004b) demonstraram que a C. benghalensis, possui em suas ceras hidrocarbonos (n-alcanos), sendo, portanto, relativamente mais hidrofóbicas, podendo influenciar na menor penetração de herbicidas hidrofílicos, como o glyphosate. Além disso, sabendoseque C. benghalensise $C$. villosa possuem quantidades semelhantes de tricomas como observado por RocHA et al. (2007). Supõe-se que a interceptação da calda de pulverização, teoricamente é idêntica para essas duas espécies, no entanto o fato de um reduzido efeito de fitointoxicação proporcionado pelo herbicida na espécie $C$. villosa pode estar relacionado com a constituição da cera, ou com o hábito de crescimento da planta, sendo que a C. villosa tem habito ereto, o que pode dificultar a interceptação das gotas pelas folhas, já a C. benghalensis tem o hábito decumbente.

Para o estudo com o corte das folhas aplicadas com os herbicidas (Tabelas 5 a 8), observa que este teve controle inferior independente do herbicida aplicado e o tempo de corte das folhas em relação ao estudo com simulação de chuva nas plantas (Tabelas 1 a 4).

Aos 7 DAA, os tratamentos apresentaram resultados semelhantes independente do produto ou tempo de corte das folhas, com exceção aos tempos 
Tabela 5 - Porcentagem de controle das plantas de Commelina villosa, aos sete dias após aplicação, em função de períodos de corte das folhas. Botucatu, SP, 2009.

\begin{tabular}{|c|c|c|c|c|c|c|c|c|c|}
\hline \multirow{2}{*}{ Tratamento } & \multirow{2}{*}{ Dose $\left(\mathrm{g} \mathrm{ha}^{-1}\right)$} & \multicolumn{8}{|c|}{ Períodos (horas) } \\
\hline & & 2 & 4 & 6 & 8 & 12 & 24 & 48 & Sem corte \\
\hline Glyphosate & 1.440 & 10,50aBC & $10,00 \mathrm{aBC}$ & $10,00 \mathrm{aBC}$ & 7,75abC & $7,50 \mathrm{bC}$ & $11,75 \mathrm{aBC}$ & $18,75 \mathrm{abAB}$ & $22,50 \mathrm{bA}$ \\
\hline $2,4-\mathrm{D}$ & 720 & $7,50 \mathrm{aBC}$ & $6,25 \mathrm{aBC}$ & $5,50 \mathrm{aC}$ & $6,50 \mathrm{bBC}$ & $10,50 \mathrm{abBC}$ & $12,50 \mathrm{aBC}$ & $15,50 \mathrm{bB}$ & $31,25 \mathrm{aA}$ \\
\hline Glyphosate+2,4-D & $1.080+720$ & $6,00 \mathrm{aD}$ & $11,00 \mathrm{aCD}$ & $11,75 \mathrm{aCD}$ & $15,00 \mathrm{aBCD}$ & $16,25 \mathrm{aBC}$ & $17,75 \mathrm{aBC}$ & $24,50 \mathrm{aB}$ & $35,00 \mathrm{aA}$ \\
\hline $\mathrm{F}_{\text {Herbicida }}(\mathrm{H})$ & & & & & $14,360^{* *}$ & & & & \\
\hline $\mathrm{F}_{\text {Chuva }}(\mathrm{C})$ & & & & & $34,949^{* *}$ & & & & \\
\hline $\mathrm{F}(\mathrm{H}) \times \mathrm{F}(\mathrm{C})$ & & & & & $2,067^{*}$ & & & & \\
\hline $\mathrm{CV}(\%)$ & & & & & 31,4 & & & & \\
\hline Dms $(H)$ & & & & & 7,33 & & & & \\
\hline Dms (C) & & & & & 9,57 & & & & \\
\hline
\end{tabular}

** significativo a $1 \%$ de probabilidade; * significativo a $5 \%$ de probabilidade. Médias seguidas de mesma letra minúscula na coluna e maiúscula na linha, não diferem estatisticamente entre si, pelo teste de tukey $(\mathrm{P}>0,05)$.

Tabela 6 - Porcentagem de controle das plantas de Commelina villosa, aos quatorze dias após aplicação, em função de períodos de corte das folhas. Botucatu, SP, 2009.

\begin{tabular}{|c|c|c|c|c|c|c|c|c|c|}
\hline \multirow{2}{*}{ Tratamento } & \multirow{2}{*}{$\begin{array}{c}\text { Dose (g } \\
\left.\text { ha }^{-1}\right)\end{array}$} & \multicolumn{8}{|c|}{ Períodos (horas) } \\
\hline & & 2 & 4 & 6 & 8 & 12 & 24 & 48 & Sem corte \\
\hline Glyphosate & 1.440 & $10,00 \mathrm{aC}$ & $12,50 \mathrm{aBC}$ & $11,25 \mathrm{bBC}$ & $25,00 \mathrm{aABC}$ & $27,50 \mathrm{aAB}$ & $30,00 \mathrm{aA}$ & $36,25 \mathrm{aA}$ & $34,00 \mathrm{bA}$ \\
\hline $2,4-\mathrm{D}$ & 720 & $11,25 \mathrm{aC}$ & $15,00 \mathrm{aBC}$ & $28,75 \mathrm{aAB}$ & $17,50 \mathrm{aBC}$ & $21,75 \mathrm{aABC}$ & $20,00 \mathrm{aABC}$ & $28,75 \mathrm{aAB}$ & $35,00 \mathrm{bA}$ \\
\hline Glyphosate+2,4-D & $1.080+720$ & $13,75 \mathrm{aB}$ & $20,00 \mathrm{aB}$ & $23,75 \mathrm{abB}$ & $24,25 \mathrm{aB}$ & $23,00 \mathrm{aB}$ & $22,50 \mathrm{aB}$ & $30,00 \mathrm{aB}$ & $67,50 \mathrm{aA}$ \\
\hline $\mathrm{F}_{\text {Herbicida }}(\mathrm{H})$ & & & & & $5,526^{* *}$ & & & & \\
\hline $\mathrm{F}_{\text {Chuva }}(\mathrm{C})$ & & & & & $22,856^{* *}$ & & & & \\
\hline $\mathrm{F}(\mathrm{H}) \times \mathrm{F}(\mathrm{C})$ & & & & & $4,630^{* *}$ & & & & \\
\hline $\mathrm{CV}(\%)$ & & & & & 30,5 & & & & \\
\hline Dms (H) & & & & & 12,7 & & & & \\
\hline Dms (C) & & & & & 16,5 & & & & \\
\hline
\end{tabular}

**significativo a 1\% de probabilidade. Médias seguidas de mesma letra minúscula na coluna e maiúscula na linha, não diferem estatisticamente entre si, pelo teste de tukey $(\mathrm{P}>0,05)$.

de 12 horas e sem corte das plantas para a aplicação do glyphosate, e para o tempo de 8 e 48 horas para a aplicação de 2,4-D (Tabela 5). Em relação aos tempos de corte das folhas tratadas, observa-se que para o tratamento onde não foi realizado o corte as plantas apresentaram um controle maior para todos os herbicidas testados, sendo que quando o glyphosate foi aplicado na forma isolada, o tratamento sem corte não diferiu estatisticamente do tempo de 48 horas de corte.

Observa-se que aos 14 DAA (Tabela 6), não foi observado diferença significativa entre glyphosate+2,4$\mathrm{D}$ e o 2,4-D isolado, exceto para o tratamento sem corte onde o efeito da mistura foi mais pronunciado.

Aos 21 DAA (Tabela 7) os menores valores de fitointoxicação foram com 2,4-D. Ressalta-se que o controle das plantas com o herbicida glyphosate aplicado na forma isolada foi superior a partir de 8 horas de intervalo para o corte das folhas, porém quando não houve o corte a mistura de glyphosate+2,4-D, foi a que obteve um maior controle (85\%). Já aos 28 DAA, observa-se que as plantas tratadas com 2,4$\mathrm{D}$ apresentaram uma diminuição no controle, isso devido a recuperação das plantas (rebrotas).
Nenhum dos tratamentos com corte das folhas proporcionou $100 \%$ de controle de C. villosa. O período de absorção do glyphosate aplicado isolado para promover controle acima de $70 \%$ foi de 12 horas, atingindo o máximo de $80,5 \%$ após 48 horas. Para o 2,4-D e a mistura glyphosate + 2,4-D ocorreu maiores controle quando as plantas não foram submetidas ao corte das folhas tratadas, sendo 45,0 e $86,3 \%$ respectivamente.

Em estudo com C. virginica RAMOs; DuRIGAN (1996) observaram que a mistura de 2,4-D + glyphosate proporcionou controles superiores em relação a aplicação de 2,4-D isolado.

OliveIRAetal. (2009), observaram queo glyphosate nas formulações de concentrado solúvel (1.170 g ha $\left.^{-1}\right)$ e de grânulos autodispersíveis em água (1.440 $\left.\mathrm{g} \mathrm{ha}^{-1}\right)$, apresentaram controle de $C$. benghalensis considerado como fraco (31 a 40\%) e aceitável (71 a $80 \%$ ), respectivamente aos 50 dias após a aplicação (DAA). Enquanto que para o 2,4-D o controle foi considerado fraco para o mesmo período. Esses resultados corroboram com os obtidos no presente trabalho para a espécie C. villosa. 
Tabela 7 - Porcentagem de controle das plantas de Commelina villosa, aos 21 dias após aplicação, em função de períodos de corte das folhas. Botucatu, SP, 2009.

\begin{tabular}{|c|c|c|c|c|c|c|c|c|c|}
\hline \multirow{2}{*}{ Tratamento } & \multirow{2}{*}{ Dose $\left(\mathrm{g} \mathrm{ha}^{-1}\right)$} & \multicolumn{8}{|c|}{ Períodos (horas) } \\
\hline & & 2 & 4 & 6 & 8 & 12 & 24 & 48 & Sem corte \\
\hline Glyphosate & 1.440 & $10,00 \mathrm{bD}$ & $25,00 \mathrm{bD}$ & $52,50 \mathrm{aBC}$ & $45,00 \mathrm{aC}$ & $65,00 \mathrm{aAB}$ & $60,00 \mathrm{aABC}$ & $77,50 \mathrm{aA}$ & $65,00 \mathrm{bAB}$ \\
\hline $2,4-\mathrm{D}$ & 720 & $11,25 b C$ & $16,25 b C$ & $25,75 b C$ & $15,00 \mathrm{bBC}$ & $22,50 \mathrm{bBC}$ & $28,75 b B C$ & $40,00 \mathrm{bAB}$ & $55,00 \mathrm{bA}$ \\
\hline Glyphosate+2,4-D & $1.080+720$ & $29,50 \mathrm{aC}$ & $42,50 \mathrm{aBC}$ & $38,75 \mathrm{abBC}$ & $33,75 b C$ & $35,00 \mathrm{bBC}$ & $47,50 \mathrm{bBC}$ & $53,75 b B$ & $82,50 \mathrm{aA}$ \\
\hline $\mathrm{F}_{\text {Herbicida }}(\mathrm{H})$ & & & & & $61,146^{* *}$ & & & & \\
\hline $\mathrm{F}_{\text {Chuva }}(\mathrm{C})$ & & & & & $39,768^{* *}$ & & & & \\
\hline $\mathrm{F}(\mathrm{H}) \times \mathrm{F}(\mathrm{C})$ & & & & & $5,639^{* *}$ & & & & \\
\hline $\mathrm{CV}(\%)$ & & & & & 21,8 & & & & \\
\hline Dms (H) & & & & & 15,03 & & & & \\
\hline Dms (C) & & & & & 19,61 & & & & \\
\hline
\end{tabular}

**significativo a 1\% de probabilidade. Médias seguidas de mesma letra minúscula na coluna e maiúscula na linha, não diferem estatisticamente entre si, pelo teste de tukey $(\mathrm{P}>0,05)$.

Tabela 8 - Porcentagem de controle das plantas de Commelina villosa, aos 28 dias após aplicação, em função de períodos de corte das folhas. Botucatu, SP, 2009.

\begin{tabular}{|c|c|c|c|c|c|c|c|c|c|}
\hline \multirow{2}{*}{ Glyphosate 2,4-D } & \multirow{2}{*}{ Dose $\left(\mathrm{g} \mathrm{ha}^{-1}\right)$} & \multicolumn{8}{|c|}{ Períodos (horas) } \\
\hline & & 2 & 4 & 6 & 8 & 12 & 24 & 48 & Sem corte \\
\hline Glyphosate & 1.440 & $10,00 \mathrm{bD}$ & $32,50 \mathrm{aC}$ & $61,25 \mathrm{aB}$ & $62,00 \mathrm{aB}$ & $70,00 \mathrm{aAB}$ & $75,00 \mathrm{aAB}$ & $80,50 \mathrm{aA}$ & $78,75 \mathrm{aA}$ \\
\hline $2,4-\mathrm{D}$ & 720 & $5,25 b B$ & $2,00 \mathrm{bB}$ & $4,25 \mathrm{cB}$ & $6,75 \mathrm{cB}$ & $15,00 \mathrm{cB}$ & $9,00 \mathrm{cB}$ & $15,00 \mathrm{cB}$ & $45,00 \mathrm{bA}$ \\
\hline Glyphosate+2,4-D & $1.080+720$ & $34,50 \mathrm{aBC}$ & $38,75 \mathrm{aBC}$ & $36,25 \mathrm{bBC}$ & $31,75 b \mathrm{~b}$ & $38,75 \mathrm{bBC}$ & $47,50 \mathrm{bBC}$ & $50,00 \mathrm{bB}$ & $86,25 \mathrm{aA}$ \\
\hline $\mathrm{F}_{\text {Herbicida }}(\mathrm{H})$ & & & & & $325,172^{* *}$ & & & & \\
\hline $\mathrm{F}_{\text {Chuva }}(\mathrm{C})$ & & & & & $57,612^{* *}$ & & & & \\
\hline $\mathrm{F}(\mathrm{H}) \times \mathrm{F}(\mathrm{C})$ & & & & & $11,979 * *$ & & & & \\
\hline $\mathrm{CV}(\%)$ & & & & & 19,0 & & & & \\
\hline Dms (H) & & & & & 12,56 & & & & \\
\hline Dms (C) & & & & & 16,38 & & & & \\
\hline
\end{tabular}

**significativo a 1\% de probabilidade. Médias seguidas de mesma letra minúscula na coluna e maiúscula na linha, não diferem estatisticamente entre si, pelo teste de tukey $(\mathrm{P}>0,05)$.

O baixo controle de 2,4-D observado corrobora com resultados encontrados por CosTA et al. (2011), onde os autores atribuem a ineficiência do 2,4-D provavelmente devido ao processo de absorção lento do herbicida, bem como, observou-se também que as plantas realizavam o abortamento das partes do caule com sintomas de fitointoxicação, como estratégia para dificultar a translocação. Assim, acredita-se os autores que para o 2,4-D seja necessário maior uniformidade de deposição de gotas sobre as plantas de trapoerabas para que ocorra controle satisfatório.

\section{CONCLUSÕES}

O controle de plantas de $C$. villosa pelo herbicida glyphosate, foi influenciado por períodos de chuva, sendo que para a aplicação do herbicida 2,4-D, ocorreu influencia apenas no período de 2 horas. Os períodos de simulação de chuva não influenciaram no controle das plantas com a mistura de glyphosate $+2,4-\mathrm{D}$.
Para o estudo com corte das folhas tratadas, todos os tratamentos independente do período para corte das folhas foram influenciados de forma negativa no controle, sendo que as plantas apresentaram rebrotas quando tratadas com o herbicida 2,4-D.

\section{REFERÊNCIAS}

COSTA, N.V.; MARTINS, D.; RODRIGUES-COSTA, A.C.P.; CARDOSO, L.A. Eficácia do glyphosate e 2,4-D no controle de espécies de trapoerabas (Commelina spp.). Bioscience Journal, v.27, n.5, p.718-728, 2011.

FORNAROLLI, D.A.; RODRIGUES, B.N.; LIMA, J. de; VALÉRIO, M.A. Influência da cobertura morta no comportamento do herbicida atrazine. In: CONGRESSO BRASILEIRO DA CIÊNCIA DAS PLANTAS DANINHAS, 21., 1997, Caxambu. Resumos. Viçosa: Sociedade Brasileira da Ciência das Plantas Daninhas, 1997. p.343.

GALLI, A.J.B. Avaliação da eficiência de glyphosate em diversos produtos no controle de Commelina virginica (trapoeraba) em citros. In: CONGRESSO BRASILEIRO 
DE HERBICIDAS E PLANTAS DANINHAS, 18., 1991, Brasília. Resumos. Brasília: SBHED, 1991. p.104-105.

MACIEL, C.D.G.; POLETINE, J.P.; AMSTALDEN, S.L.; GAZZIERO, D.L.P.; RAIMONDI, M.A.; LIMA, G.R.G.; OLIVEIRA NETO, A.M.; GUERRA, N.; JUSTINIANO, W. Misturas em tanque com glyphosate para o controle de trapoeraba, erva-de-touro e capim-carrapicho em soja RR. Revista Ceres, v.58, n.1, 2011.

MONQUERO, P.A.; CHRISTOFFOLETI, P. J.; OSUNA, M.D.; PRADO, R.A. Absorção, translocação e metabolismo do glyphosate por plantas tolerantes e suscetíveis a este herbicida. Planta Daninha, v.22, n.3, p.445-451, 2004a.

MONQUERO, P.A.; CHRISTOFFOLETI, P.J.; MATAS, J.A.; HEREDIA, A. Caracterização da superfície foliar e das ceras epicuticulares em Commelina benghalensis, Ipomoea grandifolia e Amaranthus hybridus. Planta Daninha, v.22, n.2, p.203-210, 2004 b.

MONQUERO, P.A.; CURY, J. C.; CHRISTOFFOLETI, P. J. Controle pelo glyphosate e caracterização geral da superfície foliar de Commelina benghalensis, Ipomoea hederifolia, Richardia brasiliensis e Galinsoga parviflora. Planta Daninha, v.23, n.1, p.123-132, 2005.

OLIVEIRA, A.R.; FREITAS, S.P.; VIEIRA, H.D. Controle de Commelina benghalensis, C. erecta e Tripogandra diuretica na cultura do café. Planta Daninha, v.27, n.4, p.823-830, 2009.

RAMOS, H.H.; DURIGAN, J.C. Avaliação da eficiência da mistura pronta de glyphosate $+2,4$-D no controle da Commelina virginica L. em citros. Planta Daninha, v.14, n.1, p.33-41, 1996.

ROCHA, D.C.; RODELLA, R.A.; MARTINS, D.; MACIEL, C.D.G. Efeito de herbicidas sobre quatro espécies de trapoeraba. Planta Daninha, v.25, n.2, p.359-364, 2007.

SANTOS, I.C.; MEIRA, R.M.S.A.; FERREIRA, F.A.; SANTOS, L.D.T.; MIRANDA, G.V. Caracteres anatô- micos de duas espécies de trapoeraba e a eficiência do glyphosate. Planta Daninha, v.20, n.1, p.1-8, 2002.

SHERMAN, T.D.; VAUGHN, K.C.; DUKE, S.O. Mechanism of action and resistance to herbicides. In: DUKE, S.O.

(Ed.). Herbicides Resistant Crops. Boca Raton: CRC Press, 1996. p.14-28.

SOCIEDADE BRASILEIRA DA CIÊNCIA DAS PLANTAS DANINHAS. Procedimentos para instalação, avaliação e análise de experimentos com herbicidas. Londrina: SBCPD, 1995. 42p.

SOUZA, R.T.; VELINI, E.D.; PALLADINI, L.A. Aspectos metodológicos para análise de depósitos de pulverizações pela determinação dos depósitos pontuais. Planta Daninha, v.25, n.1, p.195-202, 2007.

SULLIVAN, P.A.; DONOVAN, J. T. Interaction between glyphosate and various herbicides for broadleaved weed control. Weed Research, v.20, n.4, p.255-260, 2006.

TOLLERVEY, F.E.; FRANS, R.; PANIAGUA, O.; LARA, $\mathrm{R}$. Weed control investigations in Bolivian crops 19771978. Weed Abstract, v. 29, p. 225, 1979.

TUFFI SANTOS, L.D.; MEIRA, R.M.S.A.; SANTOS, I.QQC.; FERREIRA, F.A. Efeito do Glyphosate sobre a Morfoanatomia das Folhas e do Caule de Commelila diffusa e C. benghalensis. Planta Daninha, v.22, n.1, p.101107, 2004.

VARGAS, L.; SILVA, A.A.; BORÉM, A.; FERREIRA, F.A.; TAVARES, S.; SEDIYAMA, T. Resistência de plantas daninhas a herbicidas. Viçosa-MG: JARD, 1999. 131 p.

WILSON, A.K. Commelinaceae - a review of the distribution, biology and control of the important weeds belonging to this family. Tropical Pest Management, v.27, n.3, p.405418, 1981.

Recebido em $31 / 7 / 11$

Aceito em 28/11/12 